\title{
Co-existence of Hypertension and Pre-existing Cardiovascular Disease, Hypertension, and Pre- existing Cardiovascular Disease and Mortality in Patients on Continuous Ambulatory Peritoneal Dialysis
}

\section{Xiaoyang Wang}

Zhengzhou University

Xiaojiang Zhan

Nanchang University

Qian Zhou

Sun Yat-Sen University

Xiaoran Feng

Nanchang University

FenFen Peng

Southern Medical University

Niansong Wang

Shanghai Jiao Tong University

Yueqiang Wen

Guangzhou Medical University

Xianfeng Wu ( $\triangle$ xianfengwu2@163.com)

Shanghai Jiao Tong University https://orcid.org/0000-0001-7447-5189

Research article

Keywords: dialysis, mortality, hypertension, cardiovascular disease

Posted Date: June 22nd, 2020

DOI: https://doi.org/10.21203/rs.3.rs-35998/v1

License: (c) (i) This work is licensed under a Creative Commons Attribution 4.0 International License.

Read Full License 


\section{Abstract}

Background Little is known about the effect of co-existence of hypertension (HTN) and pre-existing cardiovascular disease (CVD), pre-existing CVD, and HTN on mortality in patients on continuous ambulatory peritoneal dialysis (CAPD).

Methods We conducted a retrospective study of 3073 incident Chinese patients on CAPD from five dialysis centers between January 1, 2005 and December 31, 2018 in a real-world setting. The primary and secondary outcomes were all-cause and CVD mortality. The association between co-existence of HTN and pre-existing CVD, pre-existing CVD, and HTN and mortality was analyzed using Cox regression models.

Results Over a median of 33.7 months of follow-up, 581 (18.6\%) patients died, with 286 (9.3\%) CVD mortality. Multinomial logistic regression showed that diabetes mellitus was associated with $6.22(95 \% \mathrm{Cl}$ 4.46 to 8.68)-time risk of co-existence of HTN and pre-existing CVD After adjusting for the confounding factors, HTN plus CVD, pre-existing CVD, and HTN groups had a higher risk of all-cause mortality (HR $3.98,95 \% \mathrm{Cl} 3.07$ to 5.17 ; HR $2.18,95 \% \mathrm{Cl} 1.27$ to 3.74 ; and HR $1.83,95 \% \mathrm{Cl} 1.47$ to 2.28 ) and CVD mortality (HR 4.68, 95\% $\mathrm{Cl} 3.27$ to 6.69 ; HR 2.11, 95\% $\mathrm{Cl} 0.96$ to 4.63 ; and $\mathrm{HR} 1.87,95 \% \mathrm{Cl} 1.37$ to 2.54 ), respectively, compared to the control group. There was no significant interaction between HTN and preexisting CVD on all-cause and CVD mortality $(\beta=0.010, P=0.973 ; \beta=0.058, P=0.892)$ in the study population.

Conclusions CAPD patients with co-existence of HTN and pre-existing CVD at the start of CAPD are at highest risk of all-cause and CVD mortality, followed by pre-existing CVD patients and HTN patients accordingly, with diabetes mellitus as a robustly predictor for co-existence of HTN and pre-existing CVD.

\section{Introduction}

Although renal replacement therapy (RRT) has been significantly improved in recent decades, the overall prognosis of end stage renal disease (ESRD) remains poor, with only $11 \%$ of peritoneal dialysis patients surviving past 10 years [1]. Cardiovascular disease (CVD) accounts for approximately $50 \%$ of deaths in dialysis patients [2]. Dialysis patients have 10 to 30 times of CVD mortality than the general population, even after adjusting for age, gender, and ethnicity [3]. Dialysis patients have a high prevalence of traditional CVD risk factors, such as hypertension (HTN), pre-existing CVD, diabetes mellitus, etc. Managing CVD risk factors is an important part of caring for dialysis patients.

Two larger peritoneal dialysis facilities in China separately reported that the presence of HTN affects $65.7 \%$ and $73.8 \%$ of patients, with $30.3 \%$ and $10.5 \%$ prevalence of pre-existing CVD, respectively $[4,5]$. Another national dialysis study of the Unite States reported that there is $72.6 \%$ prevalence of HTN, with $25.9 \%$ prevalence of coronary artery disease [6]. Previous studies reported that elevated, lower or uncontrolled BP is associated with increased mortality in the dialysis population [7-9], and dialysis patients with pre-existing CVD have poorer survival compared to those without pre-existing CVD $[6,10]$. 
Furthermore, the co-existence of HTN and pre-existing CVD is associated with a poor prognosis in the general population [11]. However, the effect of co-existence of HTN and pre-existing CVD, pre-existing CVD, and HTN on mortality to date remained unknown among dialysis patients. The objective of this study was to evaluate the association between the co-existence of HTN and pre-existing CVD, pre-existing CVD, and HTN at the start of dialysis and mortality in CAPD patients.

\section{Materials And Methods}

\section{Study Design and Population}

We conducted a retrospective cohort study of 3073 incident CAPD patients from five PD centers of three provinces in China, between January 1,2005, and December 31, 2018. To maximumly represent realworld settings of CAPD population, no patient were excluded in this study. The study was approved by the Human Ethics Committee of each research center, consistent with the ethical principles of the Declaration of Helsinki.

\section{Data Collection and Definitions}

Data on demographics, comorbid conditions, medications, and laboratory values at the start of CAPD were abstracted from medical records by two trained investigators in each center using uniform and standardized data collection tools: demographic characteristics (age, sex, body mass index, systolic BP, diastolic BP, 24-hour urine volume, current smoking, and current alcohol consumption); comorbidities (diabetes mellitus, pre-existing CVD, HTN, hyperlipidemia); medications (calcium channel blockers, beta blockers, angiotensin II receptor blockers/angiotensin-converting enzyme inhibitors [ACEI/ARBs], diuretics, statins, and aspirin); and laboratory variables (hemoglobin, serum albumin, serum uric acid, estimated glomerular filtration rate [eGFR], cholesterol, triglyceride, high density lipoprotein, low density lipoprotein, high-sensitivity C-reactive protein [hs-CRP]). The diagnosis of hypertension from 2016 Guidelines for the Management of Renal Hypertension in China was defined as systolic blood pressure $>140 \mathrm{mmHg}$ or diastolic blood pressure $>90 \mathrm{mmHg}$, or the use of antihypertensive medications [12]. The presence of CVD were defined as coronary heart disease, congestive heart failure, arrhythmias, cerebrovascular disease, or peripheral vascular disease [13]. Current smoking was defined as at least one cigarette a day,

and current alcohol consumption defined as $>20$ grams of ethanol a day [14]. eGFR was calculated using the Chronic Kidney Disease Epidemiology Collaboration equation [15].

\section{Outcomes and Follow-Up}

The primary and secondary outcomes were all-cause and CVD mortality, respectively. If the patients died in any hospital, the exact cause of death was available by death certificates, and if the patients died outside a hospital, experts would meet a consensus on the cause of death, with a comprehensive consideration of recent health conditions provided by family members, and the medical history and descriptions from each dialysis center. Patients who died within three months from transferring to hemodialysis to death were considered to receive failure of CAPD therapy and not to be censored. All 
participants were conducted CAPD schedules produced by dialysis professionals in accordance with International Standardized Peritoneal Dialysis Guidelines[16], and patient's health conditions.

All patients were followed up until CAPD cessation, death, the end of 8-year duration, or as of June 30, 2019. Transferring to hemodialysis with survival time of at least three months, renal transplantation, transferring to other centers, loss of follow-up, still survival with a follow-up period of 8 years, or as of June 30, 2019, were considered to be censored.

\section{Statistical Analysis}

Variables with missing data before the data analysis were imputed using the missForest method, coping with different types of variables [17]. Incidence was calculated as number of events divided by total valid observational time at risk, scaled to episodes per 1000 years. Variables are presented as mean \pm standard deviation (SD) or median (interquartile range, IQR) or number (\%). Patients were divided into four groups: control group (those without HTN and pre-existing CVD), CVD group (pre-existing CVD), HTN group (HTN patients), and HTN plus CVD group (patients with co-existence of HTN and pre-existing CVD). Baseline variables were compared by the One-Way ANOVA or Kruskal-Wallis tests according to variable distribution (normality tested with Shapiro-Wilk test) for quantitative variables, and the chi-square test when appropriate for categorical variables among the groups. Multinomial logistic regression was conducted to evaluate the association between baseline variables and the co-existence of HTN and pre-existing CVD, pre-existing CVD, and HTN versus the control group. The following factors were included in multinomial logistic regression, based on findings of previous studies and clinical knowledge [18-20]: age, sex, body

mass index, systolic BP, diastolic BP, current smoking, current alcohol consumption, 24-hour urine volume, diabetes mellitus, hyperlipidemia, hemoglobin, serum albumin, serum uric acid, eGFR, cholesterol, triglyceride, high density lipoprotein, low density lipoprotein, and hs-CRP.

We used Kaplan-Meier curves to investigate the difference of cumulative mortality among four groups over the observational period. To analyze the association between these interesting comorbidities and mortality, we constructed four Cox proportional hazards regression models adjusted for the following factors: Model 1, unadjusted; model 2, model 1 plus age, sex, body mass index, systolic BP, diastolic BP, current smoking, current alcohol consumption, 24-hour urine volume, diabetes mellitus, and hyperlipidemia; model 3, model 2 plus medications; model 4, model 3 plus hemoglobin, serum albumin, serum uric acid, eGFR, cholesterol, triglyceride, high density lipoprotein, low density lipoprotein, and hsCRP. In addition, the association was also analyzed among subgroups of men, women, diabetes mellitus, non-diabetes mellitus, hyperlipidemia, and non-hyperlipidemia. The interaction between HTN and preexisting CVD on all-cause and CVD mortality was examined by performing a formal test of interaction.

\section{Sensitivity Analysis}

First, for all-cause mortality, hemodialysis or renal transplants were considered competing risks. When using hemodialysis or renal transplants as competing risks, we evaluated the association between these interesting comorbidities and all-cause mortality using four Fine and Gray competing risk models. 
Similarly, for CVD mortality, non-CVD mortality, hemodialysis or renal transplants were considered competing risks. Second, as for those adult patients with a short-term period of follow-up, the interesting outcomes may not be completely observed, with under-reporting of the incidence of mortality. For fully observing outcomes, we further analyzed the effect of comorbidities at the start of dialysis on mortality in those adult patients with at least 24-month period of follow-up.

The results of the Cox proportional hazards models and the Fine and Gray models were presented as the hazard ratio (HR) and the $95 \%$ confidence interval $(\mathrm{Cl})$. Statistical analyses were conducted by GraphPad Software 8.0 (GraphPad Prism Software Inc., San Diego, California) and the R package 3.6.0 (https://www.r-project.org/). The level of significance was set as 0.05 for all analyses.

\section{Results}

\section{Patient Characteristics and Comorbidities}

All 3073 incident CAPD patients from five dialysis centers were included in the present study. All variables with less than $5 \%$ missing data were imputed before the data analysis, and there was no missing data for outcomes. Of 3073 with a median age of 49.0 (IQR 39.0-61.0), 1780 (57.9\%) were men, 1987 (64.6\%) had HTN, 431 (14.1\%) had pre-existing CVD, and 567 (18.4\%) had diabetes mellitus. All patients were divided into four group: HTN plus CVD group ( $n=370,12.0 \%)$, CVD group ( $n=60,2.0 \%), H T N(52.6 \%)$ group, and the control group ( $n=1027,33.4 \%$, Table 1). Compared to the control group, HTN plus CVD group tended to be elderly, with higher body mass index, systolic BP, hemoglobin, and cholesterol, but lower diastolic BP, as well as more likely to be current smoking, diabetes mellitus, hyperlipidemia, taking calcium channel blockers, beta blockers, diuretics, ACEI/ARBs, aspirin, and statins.

\section{Related Factors and Co-existence of HTN plus preexisting CVD, Preexisting CVD, and HTN}

We analyzed baseline factors associated with the co-existence of HTN and CVD, pre-existing CVD, and HTN versus the control group using the multinomial logistic regression (Table 2). When adjusting for confounding factors, elderly age and diabetes mellitus were associated with higher risk of co-existence of HTN and CVD, pre-existing CVD, and HTN versus the control group. Men was independently associated with higher risk co-existence of HTN and CVD, lower body mass index was associated with higher risk of pre-existing CVD, higher systolic BP and diastolic BP were associated with higher risk of HTN and coexistence of HTN and pre-existing CVD, lower hemoglobin was associated with higher risk of HTN, and higher cholesterol and lower low density cholesterol were associated with higher risk of co-existence of HTN and pre-existing CVD, compared with the control group. Of note, diabetes mellitus was associated with 6.22 (4.46 to 8.68)-time risk of co-existence of HTN and pre-existing CVD, followed by hyperlipidemia with 2.05 of odd ratio (1.51 to 2.78). Lower levels of low density cholesterol was associated with high risk of co-existence HTN and pre-existing CVD (odd ratio $0.98,95 \% \mathrm{Cl} 0.97$ to 0.99 ). 


\section{Observational Period and Mortality}

The median observational period was 33.7 (IQR 15.7-60.9) months. During this period, $571(18.6 \%, 95 \% \mathrm{Cl}$ 17.1 to $20.0 \%)$ patients died, with $286(9.3 \%, 95 \% \mathrm{Cl} 8.2 \%$ to $10.4 \%)$ CVD deaths, $59(1.9 \%, 95 \% \mathrm{Cl} 1.2 \%$ to $2.8 \%)$ infection deaths, $10(0.3 \%, 95 \% \mathrm{Cl} 0.1 \%$ to $0.6 \%)$ gastrointestinal bleeding, $17(0.6 \%, 95 \% \mathrm{Cl} 0.3$ to $0.8 \%)$ tumor deaths, $101(3.3 \%, 95 \% \mathrm{Cl} 2.7 \%$ to $3.9 \%)$ other death causes, and $89(2.9 \%, 95 \% \mathrm{Cl} 2.3 \%$ to $3.5 \%)$ unknown death causes. In addition, $375(12.2 \%, 95 \% \mathrm{Cl} 11.2 \%$ to $13.4 \%)$ transferring to hemodialysis, $159(5.2 \%, 95 \% \mathrm{Cl} 4.5 \%$ to $6.0 \%)$ receiving renal transplants, $26(0.8 \%, 95 \% \mathrm{Cl} 0.6 \%$ to $1.2 \%)$ transferring to other dialysis centers, and $106(3.4 \%, 95 \% \mathrm{Cl} 2.8 \%$ to $4.1 \%)$ loss of follow-up. The number of all-cause mortality was 143 (38.6\%, 95\% Cl 33.7\% to $44.2 \%$ ), 15 (25.0\%, 95\% Cl 14.6\% to $36.0 \%), 293$ (18.1\%, $95 \% \mathrm{Cl} 16.2 \%$ to $20.1 \%)$, and $120(11.7 \%, 95 \% \mathrm{Cl} 9.7 \%$ to $13.5 \%)$ in the HTN plus CVD, CVD, HTN, and control groups, respectively. The number of CVD mortality was $76(20.5 \%, 95 \% \mathrm{Cl} 16.5 \%$ to $25.0 \%), 7$ $(11.7 \%, 95 \% \mathrm{Cl} 4.8 \%$ to $21.1 \%), 147(9.1 \%, 95 \% \mathrm{Cl} 7.7 \%$ to $10.6 \%)$, and $56(5.5 \%, 95 \% \mathrm{Cl} 4.2 \%$ to $6.8 \%)$ in the HTN plus CVD, CVD, HTN, and control groups, respectively.

The incidence of all-cause mortality was $55.7 / 1000$ patient-years in the study population, with $27.9 / 1000$ patient-years of CVD mortality incidence (Table 3). The incidence of all-cause mortality was 131.0, 74.4, 56.1, and 32.2/1000 patient-years, and CVD mortality incidence was 69.6, 34.7, 28.2, and 15.0/1000 patient-years among the HTN plus CVD, CVD, HTN, and control groups, respectively.

\section{Comorbidities and Mortality}

Survival analysis found that the HTN plus CVD group had poorer cumulative survival $(P<0.001)$ and CVD mortality-free survival $(P=0.006)$ compared to the control group (Figure 1$)$. The association between comorbidities and mortality was evaluated by the different Cox proportional hazards regression models (Table 4). When comparing to the control group, the HTN plus CVD, CVD and HTN groups had 3.98 (95\% $\mathrm{Cl} 3.07$ to 5.17$), 2.18(95 \% \mathrm{Cl} 1.27$ to 3.74$)$, and 1.83 (95\% $\mathrm{Cl} 1.47$ to 2.28$)$-time risk of all-cause morality, and $4.68(95 \% \mathrm{Cl} 3.27$ to 6.69$), 2.11(95 \% \mathrm{Cl} 0.96$ to 4.63$)$, and $1.87(95 \% \mathrm{Cl} 1.37$ to 2.54$)$-time risk for CVD mortality compared to the control group in the model 4 , respectively. Similar trends were observed among subgroups of men, women, diabetes mellitus, non-diabetes mellitus, hyperlipidemia, and nonhyperlipidemia (Figure 2). There was no significant interaction between HTN and CVD on all-cause and CVD mortality $(\beta=0.010, P=0.973 ; \beta=0.058, P=0.892)$ in the study population.

\section{Sensitivity Analysis}

When performing competing risk analyses with hemodialysis or renal transplants as the competing risk factors, the HTN plus CVD, CVD, and HTN groups had 3.00 (95\% Cl 2.19 to 4.11), 2.03 ( $95 \% \mathrm{Cl} 1.11$ to $3.73)$, and $1.37(95 \% \mathrm{Cl} 1.07$ to 1.75$)$-time risk of all-cause morality compared to the control group, respectively, in the Fine and Gray model 4. Similarly, when using non-CVD mortality, hemodialysis or renal transplants as the competing risk factors, compared to the control group, the HTN plus CVD, CVD, and HTN groups had 3.17 ( $95 \% \mathrm{Cl} 2.03$ to 4.97 ), 2.01 (95\% 0.83 to 4.89 ), and 1.54 (95\% $\mathrm{Cl} 1.08$ to 2.18 )-time risk of all-cause morality, respectively, in the Fine and Gray model 4. 
A total of $42(1.4 \%)$ patients aged < 18 years at the start dialysis were excluded, with 6 deaths at the end study. By the end of study, $810(26.3 \%)$ adult patients were follow up less than 24 months, and 282 (9.2\%) adult patients survived for less than 24 months. The remaining 1939 (63.1\%) adult patients were follow up for at least 24 months, with $283(14.6 \%, 95 \% \mathrm{Cl} 12.9 \%$ to $16.1 \%)$ of all-cause mortality and 135 (7.0\%, $95 \% \mathrm{Cl} 5.8 \%$ to $8.1 \%$ ) of CVD mortality. We found that when comparing to the control group, the HTN plus CVD, CVD, and HTN groups had 3.53 (95\% Cl 2.32 to 4.84$), 1.73$ (95\% Cl 1.01 to 2.97), and 1.46 ( $95 \% \mathrm{Cl} 1.17$ to 1.81 )-time risk of all-cause mortality, and 4.59 (95\% $\mathrm{Cl} 2.70$ to 7.79$), 1.93$ (95\% $\mathrm{Cl} 0.88$ to 4.28), and 1.58 ( $95 \% \mathrm{Cl} 1.15$ to 2.16 )-time risk of CVD mortality in the Cox regression model 4 , respectively, among adult those with at least 24-month follow-up period.

\section{Discussion}

We found that co-existence of HTN and pre-existing CVD at baseline were more strongly associated with all-cause and CVD mortality compared to either pre-existing CVD or HTN alone, with pre-existing CVD more strongly associated with all-cause and CVD mortality than HTN. Similar trends were observed by the competing risk analysis, and among men, women, diabetes mellitus, non-diabetes mellitus, hyperlipidemia, and non-hyperlipidemia subgroups, as well as in those with at least 24-month period of follow up. In addition, diabetes mellitus was robustly independently associated with co-existence of HTN and pre-existing CVD.

In our study, the prevalence of co-existence of HTN and pre-existing CVD was $12.0 \%$. However, there to date no study reporting the predictors for co-existence of HTN and pre-existing CVD. We first reported that elderly age, diabetes mellitus, hyperlipidemia, higher systolic BP, diastolic BP, and cholesterol, and lower low density cholesterol were independently associated with higher risk of co-existence of HTN and preexisting CVD. Among these predictors for co-existence of HTN and pre-existing CVD, diabetes mellitus was the strongest predictor, followed by hyperlipidemia. Interestingly, lower levels of low density cholesterol was associated with higher risk of co-existence of HTN and pre-existing CVD, which was contradicted with clinical knowledge. The reason may be that patients with co-existence of HTN and preexisting CVD had received extensive lipid management, resulting in lower levels of low density cholesterol. More importantly, we found that patients with co-existence of HTN and pre-existing CVD were more positively associated with all-cause and CVD mortality compared to either HTN or pre-existing CVD alone in CAPD patients, and pre-existing CVD was also more positively associated with all-cause and CVD mortality than HTN. Similar findings were observed by the competing risk analysis, and among men, women, diabetes mellitus, non-diabetes mellitus, hyperlipidemia, and non-hyperlipidemia subgroups, as well as in those patients with at least 24-month follow up. These findings demonstrated that compared with those without HTN and pre-existing CVD, patients with co-existence of HTN and pre-existing CVD were at highest risk of all-cause and CVD mortality, followed by pre-existing CVD patients and HTN patients accordingly.

HTN is high prevalent and plays a significant role in the mortality of dialysis patients [21]. Previous observational studies over the past decade have confirmed the "U-shaped" or "reverse J-shaped" 
relationship between BP and mortality of dialysis patients [22-25]. On the contrary, a direct linear association between systolic BP outside the unit and all-cause mortality was observed (HR 1.26 for each $10 \mathrm{mmHg}$ higher systolic $\mathrm{BP} ; 95 \% \mathrm{Cl} 1.14$ to1.40) [26]. However, there were few study focusing on the association between HTN, as a comorbidity, and mortality of dialysis patients. In the present study, after adjusting for confounding factors, HTN patients had 1.83-time all-cause mortality and 1.87-time CVD mortality as compared to those without HTN and pre-existing CVD, and similar findings were found by sensitivity analysis and in subgroups. Meanwhile, a study of 107,922 dialysis patients from the Unite States evaluated the association between the dialysis modality and mortality, with $26.0 \%$ of coronary artery disease of new ESRD patients [6]. The HR of death was significantly greater for patients with coronary artery disease compared with those without these conditions at ESRD onset CAD (HR 1.11, $95 \% \mathrm{Cl} 1.08$ to 1.14 ). We previously conducted a study of 1068 Chinese CAPD patients, with $30.8 \%$ of preexisting CVD patients from another dialysis center. This reported that $7.0 \%$ of prior stroke CAPD patients $(\mathrm{n}=75)$ had 1.82-time risk of all-cause mortality than those without this condition. In the present study, when using those without HTN and pre-existing CVD as a reference, patients with only pre-existing CVD had 2.18-time all-cause mortality and 2.11-time CVD mortality. Also, similar results were observed by sensitivity analysis and in subgroups. HR of pre-existing CVD for all-cause mortality was significantly higher than 1.11 of HR in the above-mentioned study with 2-year follow-up period [6]. The disparities of these findings may be due to (1) different ethnicity; (2) different sample size; (3) different follow-up duration. Our findings above mentioned indicted that patients with only pre-existing CVD were at higher risk of all-cause and CVD mortality than those with only HTN, as compared to those without HTN and preexisting CVD.

In the present study, baseline demographic characteristics, medications, and laboratory parameters were unmatched among four patient categories. Compared to other three patient categories, those with HTN and pre-existing CVD were more likely to be elderly age, higher percentiles of current smoking, diabetes mellitus, taking medications, hyperlipidemia, higher levels of systolic BP, hemoglobin, and cholesterol, with lower levels of diastolic BP. Elderly age, current smoking, and diabetes mellitus had an adverse effect on the prognosis of dialysis patients [27-30]. These unmatched variables at baseline among four patient categories may affect the association between co-existence of HTN and pre-existing CVD, pre-existing CVD, HTN and mortality. Thus, although adjusting baseline variables, unmatched variables at baseline among patient subcategories may affect our findings, and we should, in the near future, conduct a cohort study with well-balanced variables at baseline to vindicate the association between these interesting comorbidities and mortality among CAPD patients.

Strengths of this study included a large sample size, population from five dialysis centers, and a detailed evaluation and adjustment for all-cause and CVD risk factors of real-world data. Several limitations should be considered. First, this was a retrospective study with potential unaccounted confounding factors and the selection biases. Although after adjusting for baseline variables, we did not draw conclusions about potential causal relationship between comorbidities and mortality. Nonetheless, fluctuations of HRs among the model 2,3 , and 4 were less than $10 \%$, suggesting three models were stable and reliable for predicting outcomes [31]. Second, one challenge was the definition of HTN. The 
optimal method to diagnose HTN in peritoneal dialysis patients is an area of controversy [32]. A recent study reported that similarly to the general populations, ambulatory BP monitoring is the gold standard method in management of HTN in peritoneal dialysis patients [33]. In our study, given the effect of ethnicity on Chinese CAPD population settings, diagnosis of HTN was based on 2016 Guidelines for the Management of Renal Hypertension in China [12]. Third, although we tried to meet a consensus of death causes, death causes were not identified in $89(2.9 \%)$ patients, which may affect the association between these interesting comorbidities and CVD mortality. Lastly, although we tried to increase the generalizability of CAPD population settings with broadly inclusion criteria, all patients were from China, suggesting our findings may lack generalization to other ethnic populations.

In conclusion, CAPD patients with co-existence of HTN and pre-existing CVD at the start of CAPD are at highest risk of all-cause and CVD mortality, followed by pre-existing CVD patients and HTN patients accordingly. In addition, diabetes mellitus is a powerful predictor for co-existence of HTN and pre-existing CVD. Our findings suggested that a combined assessment of HTN and pre-existing CVD compared with separate assessment of the two comorbidities further improved risk stratification of CAPD patients at risk of mortality.

\section{Declarations}

\section{Ethics approval and consent to participate}

The study was consistent with the ethical principles of the Declaration of Helsinki and was approved by the Human Ethics Committee of the Second Affiliated Hospital of Guangzhou Medical University, Zhujiang Hospital of Southern Medical University, Jiujiang No. 1 People's Hospital, Affiliated Sixth People's Hospital, Shanghai Jiao Tong University, The First Affiliated Hospital of Zhengzhou University, and the First Affiliated Hospital of Nanchang University. Written informed consent was obtained from all participants.

\section{Consent for publication}

All authors have approved the submitted version. All authors have agreed both to be personally accountable for the author's own contributions and to ensure that questions related to the accuracy or integrity of any part of the work, even ones in which the author was not personally involved, are appropriately investigated, resolved, and the resolution documented in the literature.

\section{Availability of data and materials}

The datasets used and/or analyzed during the current study are available from the corresponding author on reasonable request.

Competing interests 
The authors declare that they have no competing interests.

\section{Funding}

No.

\section{Authors' contributions}

$X Y$ W, contributions to the conception, and drafted the work; XR F, the acquisition of data; FF P, the acquisition of data; YQ W, the acquisition of data; NS W, contributions to the conception and design of the work; $Q, Z$, analysis and interpretation of data; $X J \mathrm{Z}$, contributions to the conception and design of the work; XF W, contributions to the conception, design of the work, and revised it. All authors have read and approved the manuscript.

\section{Acknowledgements}

We express our gratitude to all patients who participated in the study.

\section{Abbreviations}

CAPD, continuous ambulatory peritoneal dialysis; HTN, hypertension; CVD, cardiovascular disease; BP, blood pressure; ACEI/ARB, beta blockers, angiotensin II receptor blockers/angiotensin-converting enzyme inhibitors; eGFR, estimated glomerular filtration rate; hs-CRP, high-sensitivity C-reactive protein; OR, odds ratio; $\mathrm{HR}$, hazards ratio; $\mathrm{Cl}$, confidence index.

\section{References}

1. Kendrick J, Teitelbaum I. Strategies for improving long-term survival in peritoneal dialysis patients. Clin J Am Soc Nephrol. 2010;5(6):1123-31.

2. Hall YN, Chertow GM: End stage renal disease. BMJ Clin Evid 2007, 2007.

3. Jha V, Garcia-Garcia G, Iseki K, Li Z, Naicker S, Plattner B, Saran R, Wang AY, Yang CW. Chronic kidney disease: global dimension and perspectives. Lancet. 2013;382(9888):260-72.

4. Xia X, He F, Wu X, Peng F, Huang F, Yu X. Relationship between serum uric acid and all-cause and cardiovascular mortality in patients treated with peritoneal dialysis. Am J Kidney Dis. 2014;64(2):257-64.

5. Zhan X, Yang M, Chen Y, Yan C, Wang Y, Zhao Q, Chen Q, Zhang L. Relationship between serum bilirubin levels and mortality in patients on peritoneal dialysis. Ren Fail. 2019;41(1):532-9.

6. Ganesh SK, Hulbert-Shearon T, Port FK, Eagle K, Stack AG. Mortality differences by dialysis modality among incident ESRD patients with and without coronary artery disease. J Am Soc Nephrol. 
2003;14(2):415-24.

7. Udayaraj UP, Steenkamp R, Caskey FJ, Rogers C, Nitsch D, Ansell D, Tomson CR. Blood pressure and mortality risk on peritoneal dialysis. Am J Kidney Dis. 2009;53(1):70-8.

8. Stidley CA, Hunt WC, Tentori F, Schmidt D, Rohrscheib M, Paine S, Bedrick EJ, Meyer KB, Johnson HK, Zager $P G$, et al. Changing relationship of blood pressure with mortality over time among hemodialysis patients. J Am Soc Nephrol. 2006;17(2):513-20.

9. Briasoulis A, Bakris GL. The OSCAR for cardiovascular disease prevention in chronic kidney disease goes to blood pressure control. Kidney Int. 2013;83(1):20-2.

10. Wu X, Yang X, Liu X, Yi C, Guo Q, Feng X, Mao H, Huang F, Yu X. Patient Survival and Technique Failure in Continuous Ambulatory Peritoneal Dialysis Patients with Prior Stroke. Perit Dial Int. 2016;36(3):308-14.

11. Banegas JR, Ruilope LM, de la Sierra A, Vinyoles E, Gorostidi M, de la Cruz JJ, Ruiz-Hurtado G, Segura J, Rodriguez-Artalejo F, Williams B. Relationship between Clinic and Ambulatory BloodPressure Measurements and Mortality. N Engl J Med. 2018;378(16):1509-20.

12. Branch CMDAN. 2016 Guidelines for the management of renal hypertension in China. Chin Med J. 2017;97:9.

13. Wen Y, Zhan X, Wang N, Peng F, Feng X, Wu X. Monocyte/Lymphocyte Ratio and Cardiovascular Disease Mortality in Peritoneal Dialysis Patients. Mediators Inflamm. 2020;2020:9852507.

14. Tu W, Wu J, Jian G, Lori J, Tang Y, Cheng H, Wu X, Wang N. Asymptomatic hyperuricemia and incident stroke in elderly Chinese patients without comorbidities. Eur J Clin Nutr. 2019;73(10):1392402.

15. Zhang L, Wang F, Wang L, Wang W, Liu B, Liu J, Chen M, He Q, Liao Y, Yu X, et al. Prevalence of chronic kidney disease in China: a cross-sectional survey. Lancet. 2012;379(9818):815-22.

16. Woodrow G, Fan SL, Reid C, Denning J, Pyrah AN. Renal Association Clinical Practice Guideline on peritoneal dialysis in adults and children. BMC Nephrol. 2017;18(1):333.

17. Stekhoven DJ, Buhlmann P. MissForest-non-parametric missing value imputation for mixed-type data. Bioinformatics. 2012;28(1):112-8.

18. Ma L, Zhao S. Risk factors for mortality in patients undergoing hemodialysis: A systematic review and meta-analysis. Int J Cardiol. 2017;238:151-8.

19. Ishii J, Takahashi H, Kitagawa F, Kuno A, Okuyama R, Kawai H, Muramatsu T, Naruse H, Motoyama S, Matsui S, et al. Multimarker approach to risk stratification for long-term mortality in patients on chronic hemodialysis. Circ J. 2015;79(3):656-63.

20. Kim CH, Kim SJ, Lee MJ, Kwon YE, Kim YL, Park KS, Ryu HJ, Oh HJ, Han SH, Yoo TH, et al. LDL cholesterol affects clinical outcomes in incident hemodialysis patients during the early stages of dialysis. Blood Purif. 2014;38(2):131-9.

21. Agarwal R, Nissenson AR, Batlle D, Coyne DW, Trout JR, Warnock DG. Prevalence, treatment, and control of hypertension in chronic hemodialysis patients in the United States. Am J Med. 
2003;115(4):291-7.

22. Port FK, Hulbert-Shearon TE, Wolfe RA, Bloembergen WE, Golper TA, Agodoa LY, Young EW. Predialysis blood pressure and mortality risk in a national sample of maintenance hemodialysis patients. Am J Kidney Dis. 1999;33(3):507-17.

23. Zager PG, Nikolic J, Brown RH, Campbell MA, Hunt WC, Peterson D, Van Stone J, Levey A, Meyer KB, Klag MJ, et al. "U" curve association of blood pressure and mortality in hemodialysis patients. Medical Directors of Dialysis Clinic, Inc. Kidney Int. 1998;54(2):561-9.

24. Tozawa M, Iseki K, Iseki C, Takishita S. Pulse pressure and risk of total mortality and cardiovascular events in patients on chronic hemodialysis. Kidney Int. 2002;61(2):717-26.

25. Li Z, Lacson E Jr, Lowrie EG, Ofsthun NJ, Kuhlmann MK, Lazarus JM, Levin NW. The epidemiology of systolic blood pressure and death risk in hemodialysis patients. Am J Kidney Dis. 2006;48(4):60615.

26. Bansal N, McCulloch CE, Rahman M, Kusek JW, Anderson AH, Xie D, Townsend RR, Lora CM, Wright J, Go AS, et al. Blood pressure and risk of all-cause mortality in advanced chronic kidney disease and hemodialysis: the chronic renal insufficiency cohort study. Hypertension. 2015;65(1):93-100.

27. Canaud B, Tong L, Tentori F, Akiba T, Karaboyas A, Gillespie B, Akizawa T, Pisoni RL, Bommer J, Port FK. Clinical practices and outcomes in elderly hemodialysis patients: results from the Dialysis Outcomes and Practice Patterns Study (DOPPS). Clin J Am Soc Nephrol. 2011;6(7):1651-62.

28. Byrne $C$, Vernon P, Cohen JJ. Effect of age and diagnosis on survival of older patients beginning chronic dialysis. JAMA. 1994;271(1):34-6.

29. Formanek P, Salisbury-Afshar E, Afshar M. Helping Patients With ESRD and Earlier Stages of CKD to Quit Smoking. Am J Kidney Dis. 2018;72(2):255-66.

30. Rhee CM, Leung AM, Kovesdy CP, Lynch KE, Brent GA, Kalantar-Zadeh K. Updates on the management of diabetes in dialysis patients. Semin Dial. 2014;27(2):135-45.

31. Kernan WN, Viscoli CM, Brass LM, Broderick JP, Brott T, Feldmann E, Morgenstern LB, Wilterdink JL, Horwitz RI. Phenylpropanolamine and the risk of hemorrhagic stroke. N Engl J Med. 2000;343(25):1826-32.

32. Vaios V, Georgianos PI, Liakopoulos V, Agarwal R. Assessment and Management of Hypertension among Patients on Peritoneal Dialysis. Clin J Am Soc Nephrol. 2019;14(2):297-305.

33. Agarwal R, Flynn J, Pogue V, Rahman M, Reisin E, Weir MR. Assessment and management of hypertension in patients on dialysis. J Am Soc Nephrol. 2014;25(8):1630-46.

\section{Tables}

Table 1. The baseline demographic characteristics, medications, and laboratory parameters among four groups. 


\begin{tabular}{|c|c|c|c|c|c|c|}
\hline & Study population & Control group & HTN group & CVD group & HTN plus CVD group & P-value \\
\hline & 3073 & 1027 & 1616 & 60 & 370 & \\
\hline irs & $49.0(39.0-61.0)$ & $45.0(34.0-56.0)$ & $49.0(39.0-60.0)$ & $54.0(44.0-64.0)$ & $62.0(52.0-70.0)$ & $<0.001$ \\
\hline tification & & & & & & $<0.001$ \\
\hline rs & $876(28.5 \%)$ & $403(39.2 \%)$ & $436(27.0 \%)$ & $12(20.0 \%)$ & $25(6.8 \%)$ & \\
\hline years & $741(24.1 \%)$ & $253(24.8 \%)$ & $419(25.9 \%)$ & $15(25.0 \%)$ & $54(14.6 \%)$ & \\
\hline years & $665(21.7 \%)$ & $202(19.8 \%)$ & $364(22.5 \%)$ & $12(20.0 \%)$ & $87(23.5 \%)$ & \\
\hline years & $539(17.6 \%)$ & $125(12.2 \%)$ & $284(17.6 \%)$ & $14(23.3 \%)$ & $116(31.4 \%)$ & \\
\hline \multirow[t]{2}{*}{ rs } & $252(8.2 \%)$ & $43(4.2 \%)$ & $114(7.1 \%)$ & 7 (11.7\%) & $88(23.8 \%)$ & \\
\hline & $1780(57.9 \%)$ & $568(55.3 \%)$ & $957(59.2 \%)$ & $30(50.0 \%)$ & $225(60.8 \%)$ & 0.080 \\
\hline iss index, $\mathrm{kg} / \mathrm{m}^{2}$ & $22.6 \pm 7.3$ & $22.0 \pm 7.8$ & $22.9 \pm 7.6$ & $20.9 \pm 5.4$ & $23.2 \pm 3.5$ & $<0.001$ \\
\hline $\mathrm{BP}, \mathrm{mmHg}$ & $149.8 \pm 25.7$ & $143.1 \pm 26.1$ & $153.2 \pm 24.5$ & $135.9 \pm 26.8$ & $154.6 \pm 25.6$ & $<0.001$ \\
\hline : $\mathrm{BP}, \mathrm{mmHg}$ & $87.5 \pm 15.8$ & $85.3 \pm 15.8$ & $89.6 \pm 15.6$ & $84.4 \pm 15.7$ & $84.5 \pm 15.3$ & $<0.001$ \\
\hline urine volume, $\mathrm{ml}$ & $800(500-1200)$ & $800(440-1200)$ & $800(500-1200)$ & $900(400-1262)$ & $800(450-1200)$ & 0.861 \\
\hline smoking, (\%) & $310(10.1 \%)$ & $75(7.3 \%)$ & $184(11.4 \%)$ & $2(3.3 \%)$ & $49(13.2 \%)$ & $<0.001$ \\
\hline alcohol consumption, (\%) & $114(3.7 \%)$ & $34(3.3 \%)$ & $66(4.1 \%)$ & $1(1.7 \%)$ & $13(3.5 \%)$ & 0.608 \\
\hline s mellitus, (\%) & $567(18.4 \%)$ & $67(6.5 \%)$ & $318(19.7 \%)$ & $7(11.7 \%)$ & $175(47.3 \%)$ & $<0.001$ \\
\hline jidemia (\%) & $567(18.4 \%)$ & $140(13.6 \%)$ & $282(17.5 \%)$ & $20(33.3 \%)$ & $125(33.8 \%)$ & $<0.001$ \\
\hline channel blockers (\%) & $2271(73.9 \%)$ & $608(59.2 \%)$ & $1311(81.1 \%)$ & $36(60.0 \%)$ & $315(85.1 \%)$ & $<0.001$ \\
\hline ckers (\%) & $1255(40.8 \%)$ & $362(35.2 \%)$ & $695(43.0 \%)$ & $17(28.3 \%)$ & $181(48.9 \%)$ & $<0.001$ \\
\hline s (\%) & $205(6.7 \%)$ & $32(3.1 \%)$ & $123(7.6 \%)$ & $1(1.7 \%)$ & $49(13.2 \%)$ & $<0.001$ \\
\hline RBs (\%) & $1042(33.9 \%)$ & $293(28.5 \%)$ & $556(34.4 \%)$ & $19(31.7 \%)$ & $173(46.8 \%)$ & $<0.001$ \\
\hline$\%)$ & $247(8.0 \%)$ & $30(2.9 \%)$ & $128(7.9 \%)$ & $3(5.0 \%)$ & $86(23.2 \%)$ & $<0.001$ \\
\hline$\%)$ & $439(14.3 \%)$ & $73(7.1 \%)$ & $241(14.9 \%)$ & $12(20.0 \%)$ & $113(30.5 \%)$ & $<0.001$ \\
\hline obin, g/dL & $9.3 \pm 2.8$ & $9.2 \pm 2.9$ & $9.1 \pm 2.8$ & $9.1 \pm 2.2$ & $9.8 \pm 2.9$ & $<0.001$ \\
\hline lbumin, g/dL & $3.5 \pm 0.6$ & $3.5 \pm 0.6$ & $3.5 \pm 0.5$ & $3.4 \pm 0.7$ & $3.5 \pm 0.6$ & 0.750 \\
\hline Iric acid, mg/dL & $6.9 \pm 2.3$ & $7.0 \pm 2.3$ & $6.9 \pm 2.4$ & $6.4 \pm 2.2$ & $6.8 \pm 2.2$ & 0.184 \\
\hline$. \mathrm{L} / \mathrm{min} / 1.73 \mathrm{~m}^{2}$ & $6.4(4.7-8.3)$ & $6.6(4.7-8.5)$ & $6.4(4.7-8.2)$ & $6.1(4.6-8.4)$ & $6.2(4.7-8.2)$ & 0.415 \\
\hline rol, mg/dL & $151(117-183)$ & $146(112-179)$ & 153 (118-183) & $157(132-187)$ & 157 (125-187) & 0.045 \\
\hline ride, mg/dL & $94(57-153)$ & $92(62-149)$ & $95(53-156)$ & $85(32-158)$ & $99(59-153)$ & 0.413 \\
\hline nsity lipoprotein, mg/dL & $40(31-50)$ & $39(31-51)$ & $40(32-50)$ & $41(31-53)$ & $38(31-48)$ & 0.525 \\
\hline ısity lipoprotein, mg/dL & $82(48-117)$ & $82(54-118)$ & $82(4-116)$ & $89(37-120)$ & $79(30-116)$ & 0.861 \\
\hline $\mathrm{mg} / \mathrm{L}$ & $4.4(1.9-14.2)$ & $4.1(1.8-12.5)$ & $4.5(2.1-14.1)$ & $4.5(1.7-20.1)$ & $4.4(1.9-18.9)$ & 0.643 \\
\hline
\end{tabular}


Control group: patients without HTN and pre-existing CVD.

HTN, hypertension; CVD, cardiovascular disease; BP, blood pressure; ACEI/ARB, beta blockers, angiotensin II receptor blockers/angiotensinconverting enzyme inhibitors; eGFR, estimated glomerular filtration rate; hs-CRP, high-sensitivity C-reactive protein.

Table 2. Adjusted ORs for co-existence of HTN and pre-existing CVD, pre-existing CVD, and HTN using the multinomial logistic regression.

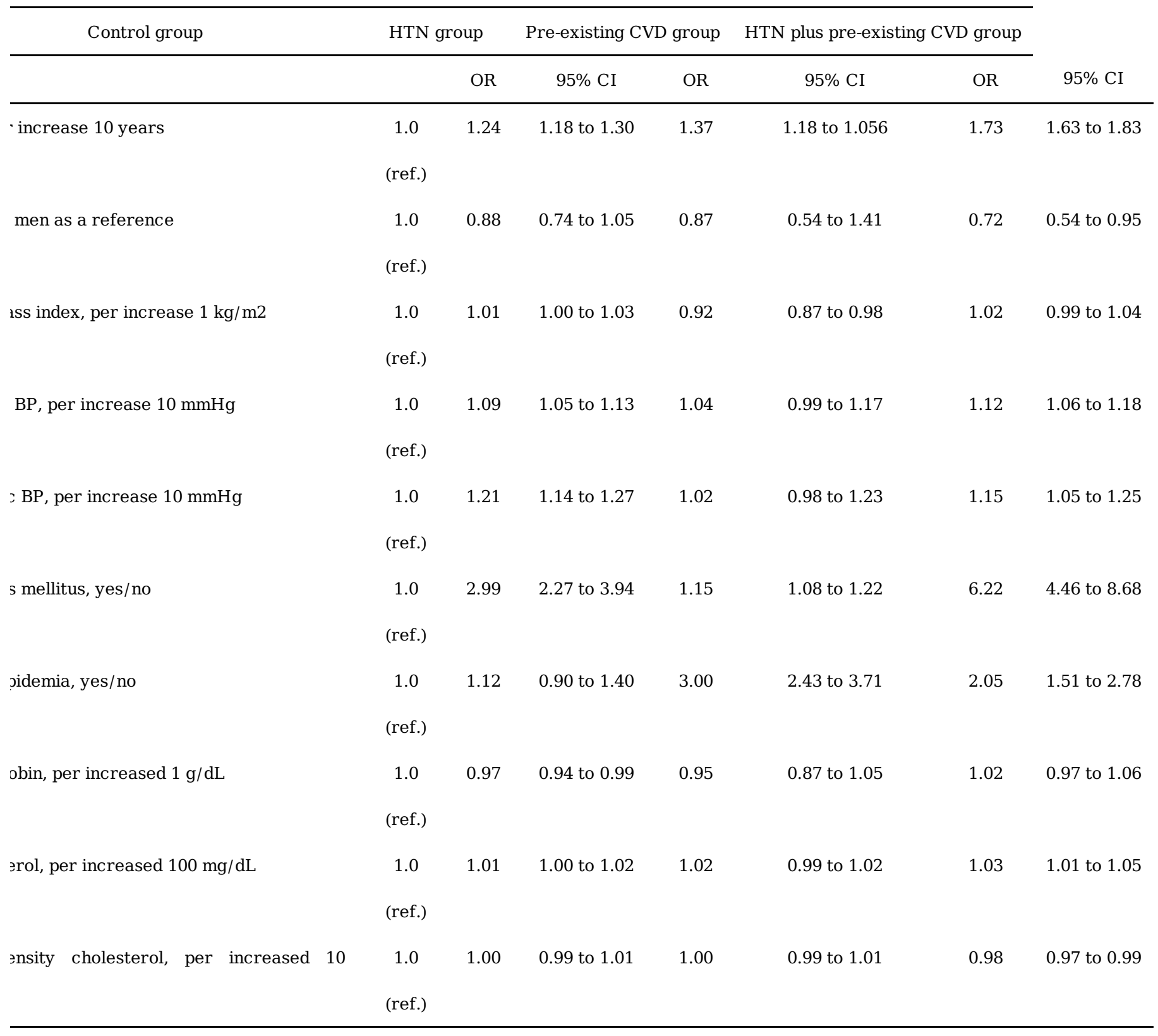

The following variables at baseline were in the multinomial logistic regression model: age, sex, body mass index, systolic BP, diastolic BP, current smoking, current alcohol consumption, 24-hour urine volume, diabetes mellitus, hyperlipidemia, hemoglobin, serum albumin, serum uric acid, eGFR, cholesterol, triglyceride, high density lipoprotein, low density lipoprotein, and hs-CRP.

HTN, hypertension; CVD, cardiovascular disease; BP, blood pressure; eGFR, estimated glomerular filtration rate; hs-CRP, high-sensitivity Creactive protein; OR, odds ratio; CI, confidence interval. 
Table 3. All-cause and CVD mortality incidence.

\begin{tabular}{|c|c|c|c|c|c|c|c|}
\hline All-cause & CVD & Time at risk & All-cause mortality incidence $(/ 1000$ & $95 \%$ & CVD mortality incidence $(/ 1000$ & $95 \%$ & \\
\hline mortality & mortality & (years) & patient-years) & CI & patient-years) & $\mathrm{CI}$ & \\
\hline Study & 571 & 286 & 10252.5 & 55.7 & 51.3 to 59.1 & 27.9 & 24.6 to \\
\hline population & & & & & & & 30.9 \\
\hline \multirow[t]{2}{*}{ Control group } & 120 & 56 & 3729.5 & 32.2 & 27.0 to 36.9 & 15.0 & 11.0 to \\
\hline & & & & & & & 18.7 \\
\hline \multirow[t]{2}{*}{ HTN group } & 293 & 147 & 5221.9 & 56.1 & 52.0 to 62.8 & 28.2 & 23.5 to \\
\hline & & & & & & & 32.8 \\
\hline \multirow[t]{2}{*}{ CVD group } & 15 & 7 & 201.5 & 74.4 & 46.5 to 112.9 & 34.7 & 9.5 to \\
\hline & & & & & & & 60.1 \\
\hline HTN plus & 143 & 76 & 1091.6 & 131.0 & 116.9 to 151.9 & 69.6 & 54.9 to \\
\hline CVD group & & & & & & & 83.7 \\
\hline
\end{tabular}

Incidence was calculated as number of events divided by total valid observational time at risk, scaled to episodes per 1000 years.

Control group: patients without HTN and pre-existing CVD.

HTN, hypertension; CVD, cardiovascular disease; CI, confidence interval.

Table 4. Adjusted HRs for mortality among different Cox proportional hazards regression models. 


\begin{tabular}{|c|c|c|c|c|c|c|c|c|}
\hline & \multicolumn{2}{|c|}{ Model 1} & \multicolumn{2}{|c|}{ Model 2} & \multicolumn{2}{|c|}{ Model 3} & \multicolumn{2}{|c|}{ Model 4} \\
\hline & $\mathrm{HR}$ & $95 \% \mathrm{CI}$ & $\mathrm{HR}$ & $95 \% \mathrm{CI}$ & HR & $95 \% \mathrm{CI}$ & $\mathrm{HR}$ & $95 \% \mathrm{CI}$ \\
\hline \multicolumn{9}{|l|}{ All-cause mortality } \\
\hline Control group & 1.0 (ref.) & & & & & & & \\
\hline HTN group & 1.74 & 1.41 to 2.15 & 1.78 & 1.43 to 2.20 & 1.83 & 1.47 to 2.27 & 1.83 & 1.47 to 2.28 \\
\hline CVD group & 2.35 & 1.38 to 4.02 & 2.18 & 1.28 to 3.74 & 2.12 & 1.24 to 3.63 & 2.18 & 1.27 to 3.74 \\
\hline HTN plus CVD group & 4.08 & 3.20 to 5.20 & 4.11 & 3.21 to 5.26 & 3.92 & 3.02 to 5.09 & 3.98 & 3.07 to 5.17 \\
\hline \multicolumn{9}{|l|}{ CVD mortality } \\
\hline Control group & 1.0 (ref.) & & & & & & & \\
\hline HTN group & 1.87 & 1.38 to 2.55 & 1.93 & 1.42 to 2.63 & 1.90 & 1.39 to 2.59 & 1.87 & 1.37 to 2.54 \\
\hline CVD group & 2.33 & 1.06 to 5.11 & 2.28 & 1.04 to 5.00 & 2.20 & 1.01 to 4.83 & 2.11 & 0.96 to 4.63 \\
\hline HTN plus CVD group & 4.65 & 3.29 to 6.57 & 4.91 & 3.47 to 6.95 & 4.68 & 3.27 to 6.69 & 4.68 & 3.27 to 6.69 \\
\hline
\end{tabular}

Model 1, unadjusted; model 2, model 1 plus age, sex, body mass index, systolic BP, diastolic BP, current smoking, current alcohol consumption, 24hour urine volume, diabetes mellitus, and hyperlipidemia; model 3, model 2 plus medications; model 4, model 3 plus hemoglobin, serum albumin, serum uric acid, eGFR, cholesterol, triglyceride, high density lipoprotein, low density lipoprotein, and hs-CRP.

Control group: participants without HTN and pre-existing CVD.

HTN, hypertension; CVD, cardiovascular disease; BP, blood pressure; eGFR, estimated glomerular filtration rate; hs-CRP, high-sensitivity Creactive protein; $\mathrm{HR}$, hazard ratio; CI, confidence interval.

Table 5. Adjusted HRs for mortality among the Fine and Gray competing risk models. 


\begin{tabular}{|c|c|c|c|c|c|c|c|c|}
\hline & \multicolumn{2}{|c|}{ Model 1} & \multicolumn{2}{|r|}{ Model 2} & \multicolumn{2}{|c|}{ Model 3} & \multicolumn{2}{|c|}{ Model 4} \\
\hline & HR & $95 \% \mathrm{CI}$ & $\mathrm{HR}$ & $95 \% \mathrm{CI}$ & HR & $95 \% \mathrm{CI}$ & $\mathrm{HR}$ & $95 \% \mathrm{CI}$ \\
\hline \multicolumn{9}{|l|}{ All-cause mortality } \\
\hline Control group & 1.0 (ref.) & & & & & & & \\
\hline HTN group & 1.57 & 1.27 to 1.95 & 1.35 & 1.06 to 1.72 & 1.41 & 1.10 to 1.80 & 1.37 & 1.07 to 1.75 \\
\hline CVD group & 2.34 & 1.31 to 4.17 & 2.22 & 1.24 to 3.97 & 2.09 & 1.15 to 3.79 & 2.03 & 1.11 to 3.73 \\
\hline HTN plus CVD group & 3.52 & 2.51 to 4.94 & 2.91 & 2.13 to 3.97 & 3.00 & 2.19 to 4.12 & 3.00 & 2.19 to 4.11 \\
\hline \multicolumn{9}{|l|}{ CVD mortality } \\
\hline Control group & 1.0 (ref.) & & & & & & & \\
\hline HTN group & 1.73 & 1.28 to 2.33 & 1.57 & 1.11 to 2.23 & 1.60 & 1.13 to 2.26 & 1.54 & 1.08 to 2.18 \\
\hline CVD group & 2.18 & 1.09 to 4.37 & 2.07 & 0.86 to 4.95 & 2.04 & 0.84 to 4.98 & 2.01 & 0.83 to 4.89 \\
\hline HTN plus CVD group & 4.40 & 2.55 to 7.58 & 3.19 & 2.04 to 4.98 & 3.17 & 2.02 to 4.98 & 3.17 & 2.03 to 4.97 \\
\hline
\end{tabular}

Model 1, unadjusted; model 2, model 1 plus age, sex, body mass index, systolic BP, diastolic BP, current smoking, current alcohol consumption, 24hour urine volume, diabetes mellitus, and hyperlipidemia; model 3, model 2 plus medications; model 4, model 3 plus hemoglobin, serum albumin, serum uric acid, eGFR, cholesterol, triglyceride, high density lipoprotein, low density lipoprotein, and hs-CRP.

Control group: participants without HTN and pre-existing CVD.

HTN, hypertension; CVD, cardiovascular disease; BP, blood pressure; eGFR, estimated glomerular filtration rate; hs-CRP, high-sensitivity Creactive protein; $\mathrm{HR}$, hazard ratio; CI, confidence interval.

\section{Figures}



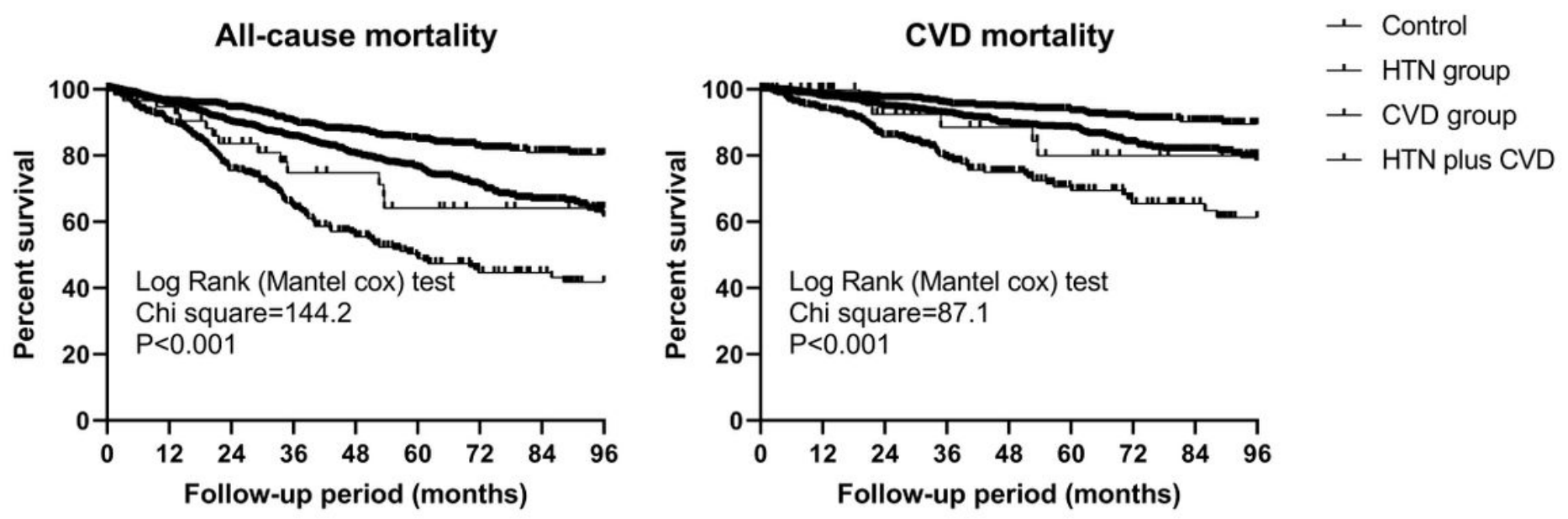

Figure 1

Cumulative survival were lowest in those with HTN and pre-existing CVD. Control group: participants without HTN and pre-existing CVD. HTN, hypertension; CVD, cardiovascular disease.
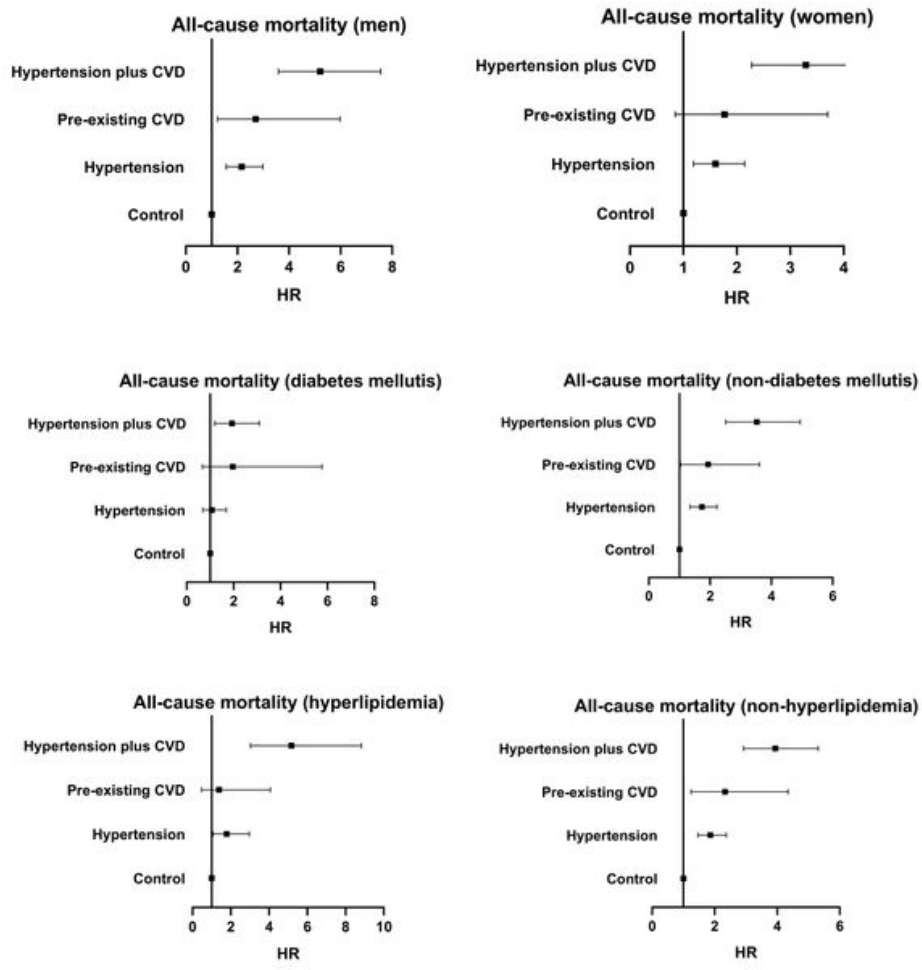
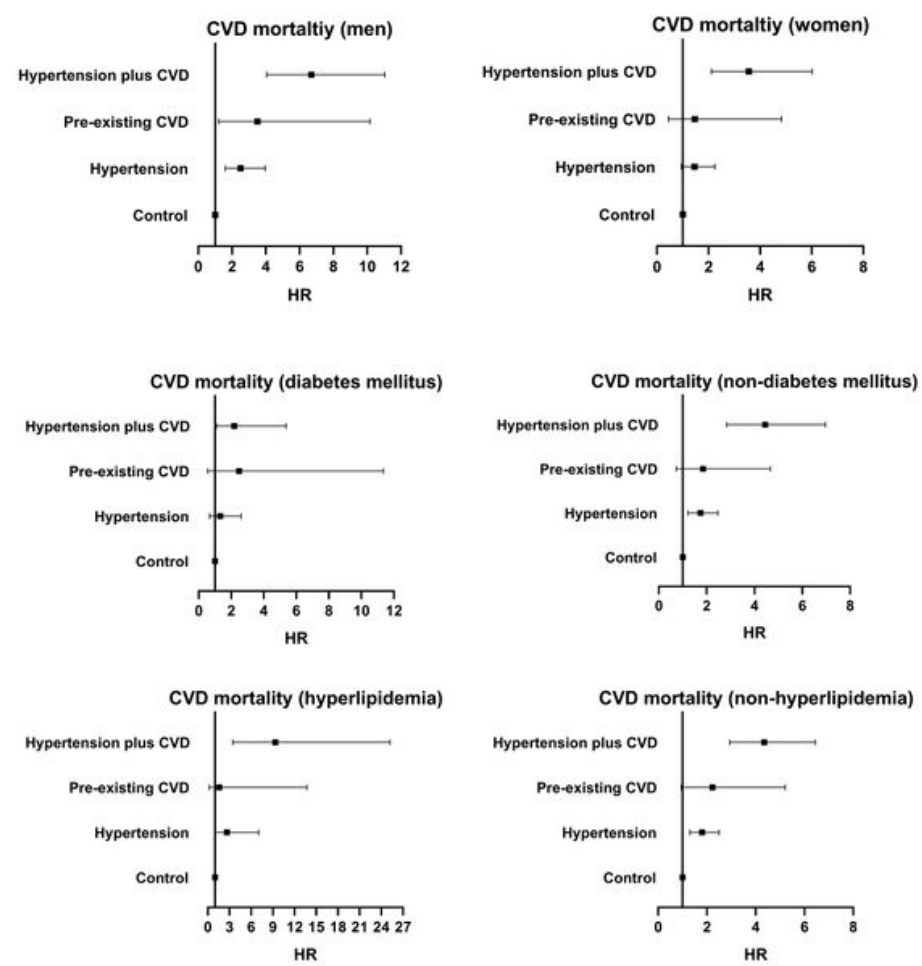

\section{Figure 2}

Adjusted HRs for all-cause and CVD mortality among subgroups. Model 1, unadjusted; model 2, model 1 plus age sex, body mass index, systolic BP, diastolic BP, current smoking, current alcohol consumption, 24-hour urine volume, diabetes mellitus, and hyperlipidemia; model 3, model 2 plus medications; model 4, model 3 plus hemoglobin, serum albumin, serum uric acid, eGFR, cholesterol, triglyceride, high density 
lipoprotein, low density lipoprotein, and hs-CRP. Control group: participants without HTN and pre-existing CVD. HTN, hypertension; CVD, cardiovascular disease; BP, blood pressure; eGFR, estimated glomerular filtration rate; hs-CRP, high-sensitivity $\mathrm{C}$-reactive protein; $\mathrm{HR}$, hazard ratio; $\mathrm{Cl}$, confidence interval. 